\title{
TarMed - Presseverlautbarungen des Konkordats Schweizer Krankenversicherer
}

\section{Sehr geehrter Herr Präsident}

Sehr geehrte Damen und Herren

Die Verbindung der Schweizer Ärztinnen und Ärzte FMH hat mit grossem Erstaunen und noch grösserem Befremden von Ihrem Pressecommuniqué Kenntnis erhalten. Wir stellen in diesem Zusammenhang fest, dass wir bis zum Abgang dieses Briefes von Ihnen weder telephonisch, postalisch, noch via Fax oder per E-mail über ihre Positionen oder Beschlüsse informiert worden sind. Wir betrachten Ihr Vorgehen als in jeglicher Hinsicht stillos, verzichten aber unsererseits ausdrücklich darauf, Verhandlungen via Medienauftritte zu führen.

In der Sache halten wir folgende Punkte fest:

1. Auf operationeller Ebene sind die Verhandlungen in den vergangenen Wochen erfolgreich verlaufen; es könnte davon ausgegangen werden, dass bis Ende April der Entwurf zu einem Rahmenvertrag, beinhaltend ein Konzept zur kostenneutralen Einführung der TarMed-Tarife, insbesondere auch zur kostenneutralen Start-Taxpunktwertberechnung in den Kantonen, vorliegen würde. Ihre mitten in der Endphase dieses Verhandlungsprozesses plakativ publizierten "Zahlen" und Überlegungen, die an dieser Stelle zu würdigen wir ausdrücklich verzichten, können nur als billiger und erpresserischer Verhandlungstrick aus der unteren Schublade bezeichnet werden.

2. Der Inhalt der sogenannten Runde 2000 wurde von Ihnen ebenso systematisch wie gegen besseres Wissen fehlinterpretiert: Sie wissen genau, dass in dieser Runde keine sogenannten "Nachverhandlungen" geführt, sondern vielmehr allseits anerkannte Inkonsistenzen, Lücken und redaktionelle Fehler bereinigt werden. Entsprechend sind die ersten Arbeiten in jeglicher Hinsicht konstruktiv verlaufen.

Es ist ein offenes Geheimnis, dass die Bewertung der operativ-interventionellen Leistungen in TarMed unzulänglich ist und einer Korrektur bedarf. Wir werden Sie separat zu Verhandlungen einladen, die dem Ziel einer Überarbeitung in der erwähnten Richtung dienen sollen.

3. Die Angelegenheit mit dem nicht beantworteten Schreiben erachteten und erachten wir als erledigt; der Präsident der Verbindung hat anlässlich des vergangenen Spitzengesprächs die notwendigen Erklärungen abgegeben und sich entschuldigt. Wir betrachten Ihr Vorgehen in dieser an und für sich nebensächlichen Angelegenheit als billig um nicht zu sagen schäbig - vielleicht auch bedenklich, weil man sich in der Tat fragt, ob solches das einzige Argumentarium ist, das Ihnen noch zur Verfügung steht.

4. Auch wir halten unverrückbar an unserer Position fest, die neuen Tarife bis 1. Januar 2001 einzuführen und seitens der FMH alle entsprechenden Voraussetzungen für die Einführung der TarMed-Tarifstrukturen in den kantonalen Tarifen zu schaffen. Wir verweisen in diesem Zusammenhang darauf, dass die Ärztekammer einen Monat vor Ihnen der Tarifstruktur Alpha 2.2 zugestimmt hat, dass die von der FMH vorzunehmenden Konzept- und Implementierungsarbeiten planmässig voranschreiten und bis heute fast 2000 ÄrztInnen in TarMed geschult wurden. Bis Ende dieses Jahres würde die gesamte Ärzteschaft geschult sein. Wir verzichten an dieser Stelle auf die Frage, wieweit die konkreten Vorbereitungsarbeiten der Versicherer gediehen sind.

Wie Sie wissen, hat Ihr wenig professionelles Vorgehen in der Ärzteschaft zu grössten Irritationen geführt. Wir können Ihnen nicht verhehlen, dass immer mehr Stimmen sogenannte "partnerschaftliche» Verhandlungen vehement in Frage stellen und die Funktion der heutigen Krankenversicherer grundsätzlich zur Diskussion bringen wollen. Die Verbindung wird Ende April eine ausserordentliche Ärztekammersitzung abhalten müssen und zum Stand der Verhandlungen mit dem KSK bzw. alternativen Vorgehensweisen Beschlüsse fassen.

Im Vorfeld dieser Ärztekammersitzung ist eine Klärung der Situation auf höchster Ebene zwischen unseren Organisationen unabdingbar; wir laden Sie zu einem Treffen am 14. April 2000, vormittags oder nachmittags, im Raume Bern-Solothurn ein. Die genaueren Modalitäten könnten zwischen den Präsidenten KSK und FMH vereinbart werden.

Mit freundlichen Grüssen

FMH

Dr. H. H. Brunner

Präsident
F.-X. Deschenaux Generalsekretär 


\section{Les vieilles traditions s'écroulent, les temps changent...}

Depuis le numéro 1 de cette année, vous pouvez lire gratuitement l'intégralité des articles du Journal Suisse de Médecine sur Internet. La homepage, créée en 1996, s'est ainsi élargie d'un service remarquable.

Voilà pour le "comment". Il reste à expliquer le "pourquoi". La planète des éditions médicales fait partie de la galaxie Gutenberg. Au premier abord, cela n'est guère compatible avec Internet. Et de plus: qui est assez idiot pour mettre gratuitement sur Internet ce qu'il vend avec succès par ailleurs?

Lorsque, l'année dernière, le directeur $d u \mathrm{Na}$ tional Institut of Health américain, le Dr Harold Varmus, a présenté le concept d'une base de données online comprenant le texte complet des travaux de recherches peer-reviewed de la biomédecine, il a déclenché une vague de réactions, pour la plupart réflexes de défense bien compréhensibles de la part des éditeurs. Les rédactions et les auteurs présentèrent aussi des arguments qualitatifs dans la discussion. Le débat "PubMed Central» est loin d'être clos.

Mais peu d'autres magazines médicaux spécialisés ont osé se jeter à l'eau. En général, les textes complets ne sont disponibles sur Internet que pour les abonnés. Mais pour combien de temps encore estil possible d'en fermer l'accès?

Mettre le contenu des journaux sur Internet et l'ouvrir à tous, de n'importe où et n'importe quand, est certainement la révolution la plus radicale de l'histoire des publications scientifiques depuis la fondation des premiers journaux peer-reviewed, il y a plus de 300 ans. Pour les auteurs, l'accès à leur secteur de recherche quelle que soit leur situation géographique est un énorme avantage. Des archives online complétées d'outils de recherche performants sont aujourd'hui l'une des raisons majeures pour consulter les pages Internet.

Une revue médicale pourvue d'un système peerreview bien conçu est un service essentiel pour les auteurs, qu'il faut perfectionner en permanence. C'est seulement ainsi que la qualité des travaux publiés peut être accrue - et ceci au profit des lecteurs.

"Because Varmus's plan is likely to get bogged down in political and commercial wrangling, we plan to press ahead with an experiment that may work because it has already worked within the high energy physics and astronomy communities" ont déjà remarqué les éditeurs du British Medical Journal en juin 1999 (Br Med J 1999;318:1637-9). Le Canadian Medical Association Journal prophétise: "Medical journals are dead. Long live medical journals" (CMAJ 2000;162:517-8).

Et Gutenberg?

Grâce à son invention, la Bible a pu être imprimée en masse, et tous ceux qui en étaient privés jusqu'alors ont pu lire ce texte fondamental. Internet pourrait jouer le même rôle pour les publications médicales. Les éditeurs médicaux sont des professionnels de la publication. Nous ne devons pas abandonner Internet à des amateurs. C'est pourquoi le Journal Suisse de Médecine est désormais online. Le Bulletin des médecins suisses va bientôt lui faire suite. Tout à fait dans la tradition Gutenberg.

Natalie Marty

EMH Editions médicales suisses SA 